\title{
Pharmacogenomics and perioperative medicine: implications for modern clinical practice
}

\author{
Ulrike M. Stamer, MD · Frank Stuber, MD
}

Received: 8 February 2009/ Accepted: 6 March 2009/Published online: 24 April 2009

(C) Canadian Anesthesiologists' Society 2009

\section{To the Editor,}

We read with interest the editorial by Kim et al. accompanying the 53-year-old publication by Werner Kalow that was reproduced from the Journal archives for the December 2008 issue. $^{1}$ The brief introduction that the authors presented regarding the impact of pharmacogenomics on the everyday care of patients is well worth reading. As implications for modern clinical practice are emphasized and clinicians are encouraged to "embrace the partnership between genetics and anesthesiology," we believe the authors should provide a specification of their comments regarding the cytochrome P-450 enzyme system CYP2D6.

Comparable to codeine, tramadol is a prodrug metabolized by hepatic cytochrome $C Y P 2 D 6$. Its M1-metabolite $(+)$-O-demethyltramadol possesses analgesic properties, and its affinity to $\mu$-opioid receptors is approximately 200 times greater than the parent compound. Thus, it is largely responsible for opioid receptor mediated analgesia, whereas (+)-tramadol and (-)-tramadol inhibit re-uptake of neurotransmitters, serotonin and noradrenaline.

CYP2D6, a highly polymorphic enzyme, is deficient in about $7-10 \%$ of Caucasians. Extensive metabolizers displaying two functional alleles present normal enzyme activity and are able to metabolize tramadol sufficiently. In previous studies, extensive metabolizers attained adequate analgesia using tramadol, in contrast to poor metabolizers

U. M. Stamer, MD ( $\square)$

University of Bonn, Bonn, Germany

e-mail: ulrike.stamer@ukb.uni-bonn.de

F. Stuber, MD

University of Bern, Inselspital, Bern, Switzerland
(PM) presenting two mutant alleles with deficient enzyme activity. $^{2}$

These PMs are characterized by deficient hydroxylation of several classes of commonly used drugs, e.g., $\beta$-blockers, antiarrhythmics, tramadol, and codeine. Patients with the PM genotype were found to be twice more likely to require additional rescue medication than patients with at least one wild-type allele. Several studies have confirmed that, compared to those genotypes carrying at least one wild-type allele, subjects carrying the PM genotype experience reduced analgesic efficacy resulting in an increased non-response rate..$^{2-4}$ Thus, the statement that "PMs may be overdosed by tramadol" ${ }^{1}$ may be misleading. Conversely, ultra-rapid CYP2D6 metabolism with an increased production of the active M1-metabolite anticipates adverse events and may result in life threatening side effects such as respiratory depression due to opioid overdose. This may occur with both prodrugs, i.e., codeine and tramadol.

Several case reports regarding codeine poisoning associated with CYP2D6 gene duplication demonstrate the pharmacogenetic impact on drug safety and patient outcome. $^{5}$ In addition to cases of respiratory depression associated with codeine, a further case has been reported regarding an ultra-rapid metabolizer receiving tramadol via patient-controlled analgesia. ${ }^{5}$ In this case, concomitant renal impairment resulting in decreased metabolite clearance enhanced opioid toxicity.

Overall, it is possible for CYP2D6 gene duplications to induce an exaggerated response to codeine and tramadol. This risk is particularly increased in patients of Ethiopian and Saudi Arabian descent due to a high prevalence of the ultra-rapid metabolizer genotype (up to $29 \%$ ).

In conclusion, there are no "opposite effects between codeine and tramadol"; essentially, there are comparable effects. However, in contrast to codeine, which does not 
modulate noradrenergic and serotonergic transmitter reuptake, patients with the PM genotype may still benefit from the monoaminergic effect of tramadol.

Conflicts of interest None declared.

\section{References}

1. Kim JH, Schwinn DA, Landau R. Pharmacogenomics and perioperative medicine-implications for modern clinical practice. Can J Anesth 2008; 55: 799-806.

2. Poulsen L, Arendt-Nielsen L, Brosen K, Sindrup SH. The hypoalgesic effect of tramadol in relation to CYP2D6. Clin Pharmacol Ther 1996; 60: 636-44.

3. Enggaard TP, Poulsen L, Arendt-Nielsen L, Brosen K, Ossig J, Sindrup $S H$. The analgesic effect of tramadol after intravenous injection in healthy volunteers in relation to CYP2D6. Anesth Analg 2006; 102: 146-50.

4. Stamer UM, Musshoff F, Kobilay M, Madea B, Hoeft A, Stuber F. Concentrations of tramadol and $O$-desmethyltramadol enantiomers in different CYP2D6 genotypes. Clin Pharmacol Ther 2007; 82: 41-7.

5. Stamer UM, Stuber F, Muders T, Musshoff F. Respiratory depression with tramadol in a patient with renal impairment and CYP2D6 gene duplication. Anesth Analg 2008; 107: 926-9.

\section{Reply}

We would like to thank Drs. Stamer and Stuber for their detailed description of the role that CYP2D6 variation has in the pharmacokinetic fate of codeine and tramadol. We agree with this description and with their assessment that codeine and tramadol display variable clinical effects for multiple reasons, including the genotype at CYP2D6 and the relative analgesic effects of parent compound versus biotransformation product. It was our intention to emphasize this clinically heterogeneous response to codeine and tramadol metabolism.

Compared to patients with extensive metabolism, poor metabolizers (PM) of tramadol may not only experience inadequate analgesia, ${ }^{1}$ but they may also have up to a $50 \%$ higher maximum concentration $\left(C_{\max }\right)$ of circulating tramadol enantiomers and an almost twofold increase in mean area under the plasma concentration-time curves after the same oral administration dose. ${ }^{2}$ This suggests that an upward titration of a PM patient's dose to achieve a desired therapeutic effect has a greater potential to yield an overdose manifested by seizures or serotonin syndrome that is related to tramadol's inhibition of norepinephrine and serotonin reuptake. In addition, tramadol should be cautiously given to patients in whom gene duplication is suspected, since they are at a higher risk of opioid-related adverse events. ${ }^{3}$ In these circumstances, genetic testing may help predict such adverse events in response to high enzymatic activity. A recent study of a North American cohort determined CYP2D6 duplication frequencies of $1.3 \%$ in Caucasians, $5.75 \%$ in African-Americans, and $2.0 \%$ in an ethnically mixed population ${ }^{4}$; however, frequencies among Ethiopians may be significantly higher. ${ }^{5}$

Conflicts of interest None declared.

\section{References}

1. Poulsen L, Arendt-Nielsen L, Brosen K, Sindrup S. The hypoalgesic effect of tramadol in relation to CYP2D6. Clin Pharmacol Ther. 1996; 60: 636-44.

2. García-Quetglas E, Azanza JR, Sadaba B, Munoz M, Gil I, Campanero MA. Pharmacokinetics of tramadol enantiomers and their respective phase I metabolites in relation to CYP2D6 phenotype. Pharmacol Res 2007; 55: 122-30.

3. Kirchheiner J, Keulen JT, Bauer S, Roots I, Brockmoller J. Effects of the CYP2D6 gene duplication on the pharmacokinetics and pharmacodynamics of tramadol. J Clin Psychopharmacol 2008; 28: 78-83.

4. Gaedigk A, Ndjountche L, Divakaran K, et al. Cytochrome P4502D6 (CYP2D6) gene locus heterogeneity: characterization of gene duplication events. Clin Pharmacol Ther 2007; 81: 242-51.

5. Ingelman-Sundberg $M$. The human genome project and novel aspects of cytochrome P450 research. Toxicol Appl Pharmacol 2005; 207: 52-6.

Jerry H. Kim, MD

Debra A. Schwinn, MD

Ruth Landau, MD

University of Washington School of Medicine, Seattle, WA, USA 